Research Article

\title{
Resolution of the Identity of the Classical Hardy Space by Means of Barut-Girardello Coherent States
}

\author{
Zouhaïr Mouayn \\ Department of Mathematics, Faculty of Sciences and Technics (M'Ghila), \\ Sultan Moulay Slimane University, BP 523, Beni Mellal, Morocco \\ Correspondence should be addressed to Zouhaïr Mouayn, mouayn@fstbm.ac.ma
}

Received 11 April 2012; Accepted 31 May 2012

Academic Editors: V. Moretti and W.-H. Steeb

Copyright (C) 2012 Zouhaïr Mouayn. This is an open access article distributed under the Creative Commons Attribution License, which permits unrestricted use, distribution, and reproduction in any medium, provided the original work is properly cited.

We construct a one-parameter family of coherent states of Barut-Girdrardello type performing a resolution of the identity of the classical Hardy space of complex-valued square integrable functions on the real line, whose Fourier transform is supported by the positive real semiaxis.

\section{Introduction}

The study of Hardy spaces, which originated during the 1910s in the setting of Fourier series and complex analysis in one variable, has over time transformed into a rich and multifaceted theory, providing basic insights into such topics as maximal functions, Hankel operators, Hilbert transforms, and wavelets analysis [1]. In physics, Hardy spaces are central in the rigged Hilbert space or Gel'fand triplet theory and play a crucial role in time-asymmetric quantum mechanics [2]. These spaces are usually involved in causality problems. Indeed, a Hardy function is important in signal processing because it may be used as signal filter [3].

In this paper, our aim is to construct an integral transform that connects the classical Hardy space $H_{+}^{2}(\mathbb{R})$ of complex-valued square integrable functions on the real line, whose Fourier transform is supported by the positive real semi-axis, with a one-parameter family of weighted Bergman spaces $\mathfrak{F}_{\sigma}(\mathbb{C})$ consisting of analytic functions on the complex plane, which are square integrable with respect to the measure $(z \bar{z})^{\sigma-1 / 2} K_{(1 / 2)-\sigma}(2 \sqrt{z \bar{z}}) d \mu(z)$ where $2 \sigma=1,2,3, \ldots, K_{v}(\cdot)$ denotes the MacDonald function and $d \mu$ is the Lebesgue measure on $\mathbb{C}$. These spaces have been considered by Barut and Girardello while introducing a class of coherent states associated with noncompact groups [4]. The constructed integral transform 
enables us to obtain a resolution of the identity of the Hardy space by means of a set of coherent states of Barut-Girardello type.

The paper is organized as follows. In Section 2, we review briefly the formalism of coherent states we will be using. Section 3 deals with some basic facts on the classical Hardy space $H_{+}^{2}(\mathbb{R})$. In Section 4 , we recall the definition of the weighted Bergman spaces $\mathfrak{F}_{\sigma}(\mathbb{C})$ as well as some of their needed properties. In Section 5, we construct a coherent state transform mapping the Hilbert space $L^{2}\left(\mathbb{R}^{+}\right)$of square integrable functions on the positive real half-line into the space $\mathfrak{F}_{\sigma}(\mathbb{C})$ and we compose it with a Fourier transform to get a new transform connecting the space $\mathfrak{F}_{\sigma}(\mathbb{C})$ with the Hardy space $H_{+}^{2}(\mathbb{R})$. In Section 6 , we deduce a set of coherent states of Barut-Girardello type by means of which a resolution of the identity of the Hardy space is achieved. Section 7 is devoted to a summary.

\section{Coherent States}

The first model of coherent states was the "nonspreading wavepacket" of the harmonic oscillator, which has been constructed by Schrödinger [5]. In suitable units, wave functions of these states are of the form

$$
\Phi_{\mathfrak{z}}(\tau):=\langle\tau \mid \mathfrak{z}\rangle=\pi^{-1 / 2} \exp \left(-\frac{1}{2} \tau^{2}+\sqrt{2} \tau \mathfrak{z}-\frac{1}{2} \mathfrak{z}^{2}-\frac{1}{2}|\mathfrak{z}|^{2}\right)
$$

for every $\tau \in \mathbb{R}$, where $\mathfrak{z} \in \mathbb{C}$ determines the mean values of coordinate $\hat{x}$ and momentum $\hat{p}$ according to $\langle\hat{x}\rangle:=\left\langle\Phi_{\mathfrak{z}}, x \Phi_{\mathfrak{z}}\right\rangle=\sqrt{2} \operatorname{Re} \mathfrak{z}$ and $\langle\hat{p}\rangle:=\left\langle\Phi_{\mathfrak{z}}, p \Phi_{\mathfrak{z}}\right\rangle=\sqrt{2} \operatorname{Im} \mathfrak{z}$. The variances $\sigma_{x}=\left\langle\hat{x}^{2}\right\rangle-\langle\hat{x}\rangle^{2}=1 / 2$ and $\sigma_{p}=\left\langle\hat{p}^{2}\right\rangle-\langle\hat{p}\rangle^{2}=1 / 2$ have equal values, so their product assumes the minimal value permitted by the Heisenberg uncertainty relation. The coherent state in (2.1) have been also obtained by Glauber [6] from the vacuum state $|0\rangle$ by means of the unitary displacement operator as

$$
\Phi_{\mathfrak{z}}=\exp \left(\mathfrak{z} A^{*}-\overline{\mathfrak{z}} A\right)|0\rangle,
$$

where $A, A^{*}$ are annihilation and creation operators defined by

$$
A=\frac{1}{\sqrt{2}}(\widehat{x}+i \widehat{p}), \quad A^{*}=\frac{1}{\sqrt{2}}(\widehat{x}-i \hat{p})
$$

Following [7], it was Iwata [8] who used the well-known expansion over the Fock basis $|n\rangle$ to give an expression of $\Phi_{\mathfrak{z}}$ as

$$
\left|\Phi_{\mathfrak{z}}\right\rangle=\left(e^{|\mathfrak{z}|^{2}}\right)^{-1 / 2} \sum_{n=0}^{+\infty} \frac{\mathfrak{z}^{n}}{\sqrt{n !}}|n\rangle
$$

Actually, various generalizations of coherent states have been proposed. Here, we will focus on a generalization of (2.4), according to a construction starting from a measure space $X$ " as a set of data" as presented in [9] or [10, pages 72-76]. Precisely, let $(X, \mu)$ be a measure space, and 
let $\mathscr{A}^{2} \subset L^{2}(X, \mu)$ be a closed subspace of infinite dimension. Let $\left\{\Psi_{n}\right\}_{n=0}^{\infty}$ be an orthogonal basis of $\mathscr{A}^{2}$ satisfying, for arbitrary $\zeta \in X$,

$$
\mathcal{N}(\zeta):=\sum_{n=0}^{\infty} \rho_{n}^{-1} \Psi_{n}(\zeta) \overline{\Psi_{n}(\zeta)}<+\infty
$$

where $\rho_{n}=\left\|\Psi_{n}\right\|_{L^{2}(X, \mu)}^{2}$. Therefore, the function

$$
\mathcal{K}(\zeta, \xi):=\sum_{n=0}^{\infty} \rho_{n}^{-1} \Psi_{n}(\zeta) \overline{\Psi_{n}(\xi)}
$$

defined on $X \times X$ is a reproducing kernel of the Hilbert space $\mathscr{A}^{2}$ so that we have $\mathcal{N}(\zeta):=$ $\mathcal{K}(\zeta, \zeta), \zeta \in X$. Let $\mathscr{\ell}$ be another (functional) Hilbert space with $\operatorname{dim} \mathscr{\ell}=\operatorname{dim} \mathcal{A}^{2}$ and $\left\{\phi_{n}\right\}_{n=0}^{\infty}$ an orthonormal basis of $\mathscr{H}$, which will play the role of a Fock basis. The coherent states labelled by points $\zeta \in X$ are defined as the ket-vectors

$$
|\zeta\rangle:=(\mathcal{N}(\zeta))^{-1 / 2} \sum_{n=0}^{\infty} \frac{\Psi_{n}(\zeta)}{\sqrt{\rho_{n}}}\left|\phi_{n}\right\rangle
$$

Now, by definition (2.7), it is straightforward to show that $\langle\zeta \mid \zeta\rangle_{\mathscr{H}}=1$ and that the coherent state transform

$$
W: \mathscr{H} \longrightarrow \mathscr{A}^{2} \subset L^{2}(X, \mu)
$$

defined by

$$
W[\phi](\zeta):=(\mathcal{N}(\zeta))^{1 / 2}\langle\zeta \mid \phi\rangle_{\mathscr{H}}
$$

is an isometry. Thus, for $\phi, \psi \in \mathscr{\ell}$, we have

$$
\begin{aligned}
\langle\phi \mid \psi\rangle_{d \mathcal{C}} & =\langle W[\phi] \mid W[\psi]\rangle_{L^{2}(X, \mu)} \\
& =\int_{X} d \sigma(\zeta) \mathcal{N}(\zeta)\langle\phi \mid \zeta\rangle\langle\zeta \mid \psi\rangle .
\end{aligned}
$$

Thereby, we have a resolution of the identity of $\mathscr{l}$ that can be expressed in Dirac's bra-ket notation as

$$
\mathbf{1}_{d \ell}=\int_{X} d \mu(\zeta) \mathcal{N}(\zeta)|\zeta\rangle\langle\zeta|
$$

where $\mathcal{N}(\zeta)$ appears as a weight function. 
For introductory papers on coherent states, we refer to [6] by Glauber for the radiation field and [11] by Arecchi et al. for atomic states. For an overview of this theory, we refer to early papers by Klauder (as [12]) and the survey of Dodonov [7] that contains a list of 451 references.

\section{The Hardy Space $H_{+}^{2}(\mathbb{R})$}

Recall that the classical Hardy space $\mathscr{\ell}\left(\Pi^{+}\right)$on the upper half of the complex plane $\Pi^{+}:=$ $\{z=x+i y, x \in \mathbb{R}, y>0\}$ consists of all functions $F(z)$ analytic on $\Pi^{+}$such that

$$
\sup _{y>0} \int_{\mathbb{R}}|F(x+i y)|^{2} d x<+\infty
$$

Any function $F(x+i y)$ has a unique boundary value $f(x)$ on the real line $\mathbb{R}$. That is,

$$
\lim _{y \rightarrow 0} F(x+i y)=f(x)
$$

which is square integrable on $\mathbb{R}$. Thus, a function $F \in \mathscr{\ell}\left(\Pi^{+}\right)$uniquely determines a function $f \in L^{2}(\mathbb{R})$. Conversely, any function $F$ can be recovered from its boundary values on the real line by means of the Cauchy integral [13] as follows:

$$
F(z)=\frac{1}{2 \pi i} \int_{\mathbb{R}} \frac{f(x)}{x-z} d x
$$

$f(x)$ being the function representing the boundary values of $F(z)$. The linear space of all functions $f(x)$ is denoted by $\mathscr{\ell}_{+}^{2}(\mathbb{R})$. Since there is one-to-one correspondence between functions in $\mathscr{l}\left(\Pi^{+}\right)$and their boundary values in $\mathscr{l}_{+}^{2}(\mathbb{R})$, we identify these two spaces. Moreover, using a Paley-Wiener theorem [14, page 175], one can characterize Hardy functions $f \in \mathscr{H}_{+}^{2}(\mathbb{R})$ by the fact that their Fourier transform

$$
\mp[f](t)=\frac{1}{\sqrt{2 \pi}} \int_{\mathbb{R}} e^{-i t x} f(x) d x
$$

is supported in $\mathbb{R}^{+}=[0,+\infty)$. That is,

$$
\mathscr{L}_{+}^{2}(\mathbb{R})=\left\{f \in L^{2}(\mathbb{R}), \mp[f](t)=0, \forall t<0\right\}
$$

This last definition of the Hardy space has been used in the context of the affine group and wavelets analysis [15]. Finally, it is well known that the Fourier transform $F$ is a linear isometry from $L^{2}(\mathbb{R})$ onto $L^{2}(\mathbb{R})$ under which the Hardy space $\mathfrak{l}_{+}^{2}(\mathbb{R})$ is mapped onto the Hilbert space $L^{2}\left(\mathbb{R}^{+}\right)$. The latter admits a complete orthonormal system given by the functions

$$
\psi_{n}^{\alpha}(t):=\left(\frac{n !}{\Gamma(n+\alpha+1)}\right)^{1 / 2} t^{(1 / 2) \alpha} e^{-(1 / 2) t} L_{n}^{(\alpha)}(t)
$$


in terms of the associated Laguerre polynomial defined by

$$
L_{n}^{(\alpha)}(t)=\sum_{k=0}^{n}(-1)^{k}\left(\begin{array}{c}
n+\alpha \\
n-k
\end{array}\right) \frac{t^{k}}{k !}, \quad \alpha>-1
$$

which leads to the expansion of functions in $L^{2}\left(\mathbb{R}^{+}, d t\right)$ with respect to the $\psi_{n}^{\alpha}(\cdot)$ (see [16]).

\section{The Bergman Space $\mathfrak{F}_{\sigma}(\mathbb{C})$}

In [4, page 51] Barut and Girardello have considered a countable set of Hilbert spaces $\mathfrak{F}_{\sigma}(\mathbb{C}), \sigma>0$ with $2 \sigma=1,2,3, \ldots$, whose elements are analytic functions $\varphi$ on $\mathbb{C}$. For each fixed $\sigma$, the inner product is defined by

$$
\langle\varphi, \psi\rangle_{\sigma}:=\int_{\mathbb{C}} \varphi(z) \overline{\psi(z)} d \mu_{\sigma}(z),
$$

with respect to the one-parameter measure

$$
d \mu_{\sigma}(z):=\frac{2}{\pi \Gamma(2 \sigma)} \rho^{2 \sigma-1} K_{(1 / 2)-\sigma}(2 \rho) \rho d \theta d \rho
$$

where $z=\rho e^{i \theta} \in \mathbb{C}$ and the MacDonald function [17, page 183]

$$
K_{v}(w)=\frac{1}{2}\left(\frac{w}{2}\right)^{v} \int_{0}^{+\infty} t^{-v-1} \exp \left(-t-\frac{w^{2}}{4 t}\right) d t
$$

is defined for $\operatorname{Re}\left(w^{2}\right)>0$. Precisely, $\mathfrak{F}_{\sigma}(\mathbb{C})$ consists of entire functions $\varphi$ with finite norm $\|\varphi\|_{\sigma}=\sqrt{\langle\varphi, \varphi\rangle_{\sigma}}<+\infty$. Note also that if $\varphi(z)$ is an entire function with power series $\sum_{n} c_{n} z^{n}$, then the norm square in terms of the expansion coefficients is given by

$$
\|\varphi\|_{\sigma}^{2}=(\Gamma(2 \sigma))^{-1} \sum_{n=0}^{+\infty} c_{n} \overline{c_{n}} n ! \Gamma(2 \sigma+n)
$$

An orthonormal basis function of $\mathfrak{F}_{\sigma}(\mathbb{C})$ is given by

$$
\varphi_{n}^{\sigma}(z):=\sqrt{\frac{\Gamma(2 \sigma)}{n ! \Gamma(2 \sigma+n)}} z^{n}
$$

for every $z \in \mathbb{C}$ and varying $n=0,1,2, \ldots$ 
Lemma 4.1. Let $2 \sigma=1,2,3, \ldots$. Then, the diagonal function of the reproducing kernel of the Hilbert space $\mathfrak{F}_{\sigma}(\mathbb{C})$ can be expressed in terms of the modified Bessel function as

$$
\mathcal{K}_{\sigma}(z, z)=\Gamma(2 \sigma)(z \bar{z})^{(1 / 2)-\sigma} I_{2 \sigma-1}(2 \sqrt{z \bar{z}})
$$

for every $z \in \mathbb{C}$.

Proof. By the general theory [18], the reproducing kernel of $\mathfrak{F}_{\sigma}(\mathbb{C})$ can be obtained form the orthonormal basis in (4.5) as

$$
\mathcal{K}_{\sigma}(z, w)=\sum_{n=0}^{+\infty} \varphi_{n}^{\sigma}(z) \overline{\varphi_{n}^{\sigma}(w)}
$$

Replacing the $\varphi_{n}^{\sigma}(z)$ by their expressions, then the sum in (4.7) reads

$$
K_{\sigma}(z, w)=\sum_{n=0}^{+\infty} \frac{1}{(2 \sigma)_{n}} \frac{(z \bar{w})^{n}}{n !}={ }_{0} \digamma_{1}(2 \sigma ; z \bar{w})
$$

Here, we recall the confluent hypergeometric limit function [17, page 100]:

$$
{ }_{0} \digamma_{1}(a ; x)=\sum_{n=0}^{+\infty} \frac{1}{(a)_{n}} \frac{x^{n}}{n !}
$$

in which $(a)_{n}$ denotes the Pochhammer symbol defined by $(a)_{0}:=1$ and

$$
(a)_{n}:=a(a+1) \cdots(a+j-1)=\frac{\Gamma(a+n)}{\Gamma(a)} .
$$

Making use of the relation [17, page 77]

$$
{ }_{0} \digamma_{1}\left(v+1 ;-\frac{1}{4} u^{2}\right)=\Gamma(v+1)\left(\frac{1}{2} u\right)^{-v} J_{v}(u)
$$

$J_{v}(\cdot)$ being the Bessel function of order $v \in \mathbb{R}$, and recalling the definition of the modified Bessel function of the first kind

$$
I_{\nu}(u)=\exp \left(-\frac{1}{2} v \pi i\right) J_{v}\left(e^{(1 / 2) \pi i} u\right)
$$

we obtain, for $v=2 \sigma-1, u=2 i|z|$, and $z=w$, the expression of the diagonal function of the reproducing kernel as in (4.6). 


\section{A Coherent State Transform}

We are now in a position to apply the formalism in Section 2 in order to define a set of coherent states for the data of $\left((X, d \mu), \mathscr{A}^{2},\left\{\Psi_{n}\right\}\right)$ and $\left(\mathscr{\ell},\left\{\phi_{n}\right\}\right)$ where

(i) $(X, d \mu(\zeta)) \equiv\left(\mathbb{C}, d \mu_{\sigma}(z)\right), \zeta \equiv z$ and $d \mu_{\sigma}$ is the measure defined in (4.2),

(ii) $\mathscr{A}^{2} \equiv \mathfrak{F}_{\sigma}(\mathbb{C})$ is the weighed Bergman space defined in Section 4,

(iii) $\left\{\Psi_{n}\right\} \equiv\left\{\varphi_{n}^{\sigma}(z)\right\}$ is the orthonormal basis in (4.5),

(iv) $\mathscr{l} \equiv L^{2}\left(\mathbb{R}^{+}, d t\right)$,

(v) $\left\{\phi_{n}\right\} \equiv\left\{\psi_{n}^{\alpha}\right\}$ is the orthonormal basis in (3.6) with $\alpha=2 \sigma-1$.

Definition 5.1. For each fixed $2 \sigma=1,2, \ldots$, a set of coherent states labelled by points $z \in \mathbb{C}$ and belonging to the Hilbert space $L^{2}\left(\mathbb{R}^{+}\right)$can be defined according to (2.7) through the ketvectors

$$
|z, \sigma\rangle:=\left(\mathcal{N}_{\sigma}(z)\right)^{-1 / 2} \sum_{n=0}^{\infty} \varphi_{n}^{\sigma}(z)\left|\psi_{n}^{2 \sigma-1}\right\rangle
$$

where the normalizing factor has the expression

$$
\mathcal{N}_{\sigma}(z)=\Gamma(2 \sigma)(z \bar{z})^{(1 / 2)-\sigma} I_{2 \sigma-1}(2 \sqrt{z \bar{z}})
$$

which is the quantity $\mathcal{K}_{\sigma}(z, z)$ given in Lemma 4.1.

Proposition 5.2. The coherent state transform (CST) associated with the coherent states in (5.1) is the isometry $W_{\sigma}: L^{2}\left(\mathbb{R}^{+}\right) \rightarrow \mathfrak{F}_{\sigma}(\mathbb{C})$ defined by

$$
W_{\sigma}[\psi](z)=\sqrt{\Gamma(2 \sigma)} z^{-\sigma+1 / 2} e^{z} \int_{0}^{+\infty} J_{2 \sigma-1}(2 \sqrt{z t}) \overline{\psi(t)} e^{-(1 / 2) t} d t
$$

for every $\psi \in L^{2}\left(\mathbb{R}^{+}, d t\right)$ and $z \in \mathbb{C}$.

Proof. According to (2.9), the coherent state transform $W_{\sigma}[\psi]$ of an arbitrary function $\psi \in$ $L^{2}\left(\mathbb{R}^{+}, d t\right)$ is defined by

$$
W_{\sigma}[\psi](z)=\left(\mathcal{N}_{\sigma}(z)\right)^{1 / 2}\langle z, \sigma \mid \psi\rangle_{L^{2}\left(\mathbb{R}^{+}\right)} .
$$

We make use of (5.1) to rewrite (5.4) as

$$
W_{\sigma}[\psi](z)=\left\langle\sum_{n=0}^{\infty} \varphi_{n}^{\sigma}(z) \mid \psi_{n}^{2 \sigma-1}\right\rangle|\psi\rangle_{L^{2}\left(\mathbb{R}^{+}\right)}
$$


Note that (5.5) can also be presented as

$$
W_{\sigma}[\psi](z)=\int_{0}^{+\infty} W_{\sigma}(z, t) \overline{\psi(t)} d t
$$

in terms of the kernel function

$$
W_{\sigma}(z, t):=\sum_{n=0}^{\infty} \varphi_{n}^{\sigma}(z) \psi_{n}^{2 \sigma-1}(t)
$$

Replacing $\varphi_{n}^{\sigma}(z)$ and $\psi_{n}^{2 \sigma-1}(t)$ by their expressions in (4.5) and (3.6), respectively, then (5.7) takes the form

$$
W_{\sigma}(z, t)=\sqrt{\Gamma(2 \sigma)} t^{\sigma-1 / 2} e^{-(1 / 2) t} \sum_{n=0}^{\infty} \frac{z^{n}}{\Gamma(2 \sigma+n)} L_{n}^{(2 \sigma-1)}(t)
$$

Next, we make use of the formula [19, page 1002]

$$
\sum_{n=0}^{\infty} \frac{z^{n}}{\Gamma(\beta+1+n)} L_{n}^{(\beta)}(t)=(z t)^{-((1 / 2) \beta)} e^{z} J_{\beta}(2 \sqrt{z t}), \quad \beta>-1
$$

for $\beta=2 \sigma-1$. Therefore, (5.8) becomes

$$
W_{\sigma}(z, t)=\sqrt{\Gamma(2 \sigma)} z^{-\sigma+(1 / 2)} e^{z} e^{-(1 / 2) t} J_{2 \sigma-1}(2 \sqrt{z t}) .
$$

Finally, inserting the last expression of $W_{\sigma}(z, t)$ into (5.6), we arrive at the announced result.

Now, with the help of the CST $W_{\sigma}$ we construct the following integral transform.

Theorem 5.3. Let $2 \sigma=1,2,3, \ldots$ be a fixed parameter. Then, the integral transform $T_{\sigma}: \mathscr{L}_{+}^{2}(\mathbb{R}) \rightarrow$ $\mathfrak{F}_{\sigma}(\mathbb{C})$ defined by composing the CST $W_{\sigma}$, with the Fourier transform $\mathcal{F}$ as $T_{\sigma}:=W_{\sigma} \circ \mathcal{F}$ is an isometric map having the explicit form

$$
T_{\sigma}[f](z)=\frac{\Gamma(\sigma+1 / 2) e^{z}}{\sqrt{2 \pi \Gamma(2 \sigma)}} \int_{\mathbb{R}}\left(\frac{1}{2}-i x\right)^{-\sigma-(1 / 2)}{ }_{1} \digamma_{1}\left(\sigma+\frac{1}{2}, 2 \sigma, \frac{-z}{(1 / 2)-i x}\right) \overline{f(x)} d x,
$$

for every $f \in \mathscr{L}_{+}^{2}(\mathbb{R})$ and $z \in \mathbb{C}$, where ${ }_{1} \digamma_{1}$ is the confluent hypergeometric function. 
Proof. Let $f \in \mathscr{H}_{+}^{2}(\mathbb{R})$. Then, we have successively

$$
\begin{aligned}
T_{\sigma}[f](z) & =\left(W_{\sigma} \circ \mathcal{F}\right)[f](z)=W_{\sigma}[\mathcal{F}[f]](z) \\
& =\sqrt{\Gamma(2 \sigma)} z^{-\sigma+(1 / 2)} e^{z} \int_{0}^{+\infty} e^{-(1 / 2) t} J_{2 \sigma-1}(2 \sqrt{z t}) \overline{\mathcal{F}[f](t)} d t \\
& =\sqrt{\Gamma(2 \sigma)} z^{-\sigma+1 / 2} e^{z} \int_{0}^{+\infty} e^{-(1 / 2) t} J_{2 \sigma-1}(2 \sqrt{z t})\left(\frac{1}{\sqrt{2 \pi}} \int_{\mathbb{R}} e^{i t x} \overline{f(x)} d x\right) d t
\end{aligned}
$$

Changing the order of integration, we rewrite (5.14) as

$$
T_{\sigma}[f](z)=\frac{\sqrt{\Gamma(2 \sigma)}}{\sqrt{2 \pi}} z^{-\sigma+1 / 2} e^{z} \int_{\mathbb{R}} T_{\sigma}(z, x) \overline{f(x)} d x
$$

where we have introduced the integral

$$
\begin{aligned}
T_{\sigma}(z, x) & :=\int_{0}^{+\infty} e^{-(1 / 2-i x) t} J_{2 \sigma-1}(2 \sqrt{z t}) d t \\
& =2 \int_{0}^{+\infty} u e^{-(1 / 2-i x) u^{2}} J_{2 \sigma-1}(2 \sqrt{z} u) d u
\end{aligned}
$$

Next, we make appeal to the identity [19, page 706]

$$
\int_{0}^{+\infty} u^{\varrho} e^{-\alpha u^{2}} J_{v}(\beta u) d u=\frac{2 \beta^{v} \Gamma((1 / 2) v+(1 / 2) \rho+1 / 2)}{2^{v+1} \alpha^{(1 / 2)(\rho+v+1)} \Gamma(v+1)} \cdot{ }_{1} \digamma_{1}\left(\frac{\rho+v+1}{2}, v+1, \frac{-\beta^{2}}{4 \alpha}\right)
$$

$\operatorname{Re} \alpha>0, \operatorname{Re}(\rho+v)>-1$ for the parameters $\rho=1, \alpha=(1 / 2)-i x, v=2 \sigma-1$, and $\beta=2 \sqrt{z}$. Here,

$$
{ }_{1} \digamma_{1}(a, b ; u)=\sum_{n=0}^{+\infty} \frac{(a)_{n}}{(b)_{n}} \frac{u^{n}}{n !}
$$

is the confluent hypergeometric function [19, page 1023]. Therefore, the integral in (5.16) reads

$$
T_{\sigma}(z, x)=\frac{\Gamma(\sigma+(1 / 2)) z^{\sigma-(1 / 2)}}{\Gamma(2 \sigma)((1 / 2)-i x)^{\sigma+(1 / 2)}} \cdot{ }_{1} \digamma_{1}\left(\sigma+\frac{1}{2}, 2 \sigma, \frac{-z}{(1 / 2)-i x}\right) .
$$

Returning back to (5.16) and replacing $T_{\sigma}(z, x)$ by its expression (5.20), we arrive at the result (5.11). 


\section{A Resolution of the Identity of $\mathfrak{l}_{+}^{2}(\mathbb{R})$}

Now, observe that if one starts with coherent states whose wave functions are expressed in a closed form, then one will be able to construct an isometric map between two functional Hilbert spaces $\mathscr{A}^{2}$ and $\mathscr{t}$ as discussed in Section 2. Here, we will take the opposite direction in the sense that we proceed to extract a set of coherent states of Barut-Girardello type belonging to the Hardy space $H_{+}^{2}(\mathbb{R})$ from the expression of the isometric map $T_{\sigma}$ in (5.11). We precisely establish the following result.

Theorem 6.1. Let $2 \sigma=1,2,3, \ldots$ be a fixed parameter. Then, the states $|w, \sigma\rangle$ labelled by points $w \in \mathbb{C}$ and defined by

$$
|w, \sigma\rangle:=\left(\mathcal{N}_{\sigma}(w)\right)^{-1 / 2} \sqrt{\frac{\Gamma(2 \sigma)}{2 \pi}} z^{-\sigma+(1 / 2)} e^{z} T_{\sigma}(w, \cdot)
$$

with the wave functions

$$
\langle x \mid w, \sigma\rangle=\frac{{ }_{1} \digamma_{1}\left(\sigma+1 / 2,2 \sigma,(i x-1 / 2)^{-1} w\right) e^{w} \Gamma(\sigma+1 / 2)}{\Gamma(2 \sigma) \sqrt{2 \pi(w \bar{w})^{1 / 2-\sigma} I_{2 \sigma-1}(2 \sqrt{w \bar{w}})}((1 / 2)-i x)^{\sigma+1 / 2}}
$$

are coherent states of Barut-Girardello type in the classical Hardy space and satisfy the following resolution of the identity:

$$
\mathbf{1}_{\mathscr{L}_{+}^{2}(\mathbb{R})}=\Gamma(2 \sigma) \int_{\mathbb{C}} d \mu(w)(w \bar{w})^{1 / 2-\sigma} I_{2 \sigma-1}(2 \sqrt{w \bar{w}})|w, \sigma\rangle\langle w, \sigma|
$$

Proof. We first make use of the relation [16, page 349]

$$
{ }_{1} \digamma_{1}\left(-a, b,\left(\frac{1-c}{c}\right) u\right) e^{u}=\sum_{j=0}^{+\infty} M_{j}(a, b, c) \frac{u^{j}}{j !}
$$

for the parameters $u=w, a=-(\sigma+(1 / 2)), \quad b=2 \sigma$, and $c=(i x-(1 / 2))((1 / 2)+i x)^{-1}$, where $M_{j}(a, b, c)$ denotes the Meixner polynomial. Therefore, the confluent hypergeometric function occurring in (6.2) can be expanded into the following series:

$$
{ }_{1} \digamma_{1}\left(\sigma+\frac{1}{2}, 2 \sigma, \frac{-w}{(1 / 2)-i x}\right) e^{w}=\sum_{j=0}^{+\infty} M_{j}\left(-\sigma-\frac{1}{2}, 2 \sigma, \frac{i x-(1 / 2)}{i x+(1 / 2)}\right) \frac{w^{j}}{j !} .
$$

Next, we write the Meixner polynomial in terms of a terminating Gauss hypergeometric ${ }_{2} \digamma_{1}-$ sum [16, page 346$]$ :

$$
M_{j}(a, b, c)={ }_{2} \digamma_{1}\left(-j,-a, b ; 1-\frac{1}{c}\right) .
$$


Therefore, (6.5) takes the form

$$
{ }_{1} \digamma_{1}\left(\sigma+\frac{1}{2}, 2 \sigma, \frac{-w}{(1 / 2)-i x}\right) e^{w}=\sum_{j=0}^{+\infty} \frac{w^{j}}{j !} \cdot{ }_{2} \digamma_{1}\left(-j, \sigma+\frac{1}{2}, 2 \sigma, \frac{1}{(1 / 2)-i x}\right) .
$$

Taking into account (6.7), the wave function in (6.2) can be rewritten as

$$
\langle x \mid w, \sigma\rangle=\left(\mathcal{N}_{\sigma}(w)\right)^{-1 / 2} \sum_{j=0}^{+\infty} \sqrt{\frac{\Gamma(2 \sigma)}{j ! \Gamma(2 \sigma+j)}} w^{j} \phi_{j}^{\sigma}(x)
$$

in terms of the functions

$$
\phi_{j}^{\sigma}(x):=\frac{\Gamma(\sigma+1 / 2)}{\sqrt{2 \pi \Gamma(2 \sigma)}} \sqrt{\frac{\Gamma(2 \sigma+j)}{j ! \Gamma(2 \sigma)}}\left(\frac{1}{2}-i x\right)^{-(\sigma+1 / 2)} \cdot{ }_{2} \digamma_{1}\left(-j, \sigma+\frac{1}{2}, 2 \sigma, \frac{1}{(1 / 2)-i x}\right) .
$$

To check that $\phi_{j}^{\sigma} \in \mathscr{\ell}_{+}^{2}(\mathbb{R})$, one can use the fact [3, Prop.1] that a function of moderate decrease is of class Hardy if and only if all its poles lie in the lower half-plane. The orthogonality of the functions $\left(\phi_{j}^{\sigma}\right)$ can be deduced from the orthogonality relations of the Laguerre polynomials by applying the integral representation

$$
{ }_{2} \digamma_{1}\left(-j, \beta+1, \alpha+1, \frac{1}{s}\right)=s^{\beta+1} \frac{j ! \Gamma(\alpha+1)}{\Gamma(\beta+1) \Gamma(\alpha+j+1)} \int_{0}^{+\infty} e^{-s t} t^{\beta} L_{j}^{(\alpha)}(t) d t,
$$

$\operatorname{Re} \beta>-1, \operatorname{Re} s>0$, for the parameters $\alpha=2 \sigma-1, \beta=\sigma-1 / 2$, and $s=(1 / 2)-i x$. In any way, if we use the Legendre duplication formula [19, page 896]

$$
\sqrt{\pi} \Gamma(2 \epsilon)=2^{2 \epsilon-1} \Gamma(\epsilon) \Gamma\left(\epsilon+\frac{1}{2}\right)
$$

satisfied by the gamma function, then the constant in (6.9) reads

$$
\frac{\Gamma(\sigma+1 / 2)}{\sqrt{2 \pi \Gamma(2 \sigma)}}=\frac{1}{2^{\sigma} \pi^{1 / 4}}\left(\frac{\Gamma(\sigma+1 / 2)}{\Gamma(\sigma)}\right)^{1 / 2}
$$

and, therefore, one can verify by [20, page 62] that the functions $\left(\phi_{j}^{\sigma}\right)$ in (6.9) written in terms of the constant in the right-hand side of (6.12) constitute a complete orthonormal system of rational functions in the Hardy space $\mathfrak{L}_{+}^{2}(\mathbb{R})$. Finally, the resolution of the identity in (6.3) follows by a direct application of (2.11). This ends the proof.

\section{Summary}

We have constructed an integral transform that connects the Hardy space $H_{+}^{2}(\mathbb{R})$ of complexvalued square integrable functions on the real line, whose Fourier transform is supported 
by the positive real semiaxis, with a one-parameter family of weighted Bergman spaces $\mathfrak{F}_{\sigma}(\mathbb{C})$ consisting of analytic functions on the complex plane, which are square integrable with respect to a measure involving the MacDonald function. These spaces are attached to the socalled Barut-Girardello coherent states. The constructed integral transform has enabled us to construct a set of coherent states that satisfy the resolution of the identity of the Hardy space.

\section{Acknowledgment}

The author is very grateful for Professor Josef Wichmann for sending him some remarks on the preprint version of this work.

\section{References}

[1] E. M. Stein, Harmonic Analysis: Real-Variable Methods, Orthogonality, and Oscillatory Integrals, Princeton University Press, Princeton, NJ, USA, 1993.

[2] R. C. Bishop, A. Bohm, and M. Gadella, "Irreversibility in quantum mechanics," Discrete Dynamics in Nature and Society, vol. 2004, no. 1, pp. 75-83, 2004.

[3] L. B. Pierce, "Hardy functions," http://people.maths.ox.ac.uk/piercel/theses/Pierce_Junior_Paper .pdf.

[4] A. O. Barut and L. Girardello, "New "coherent" states associated with non-compact groups," Communications in Mathematical Physics, vol. 21, pp. 41-55, 1971.

[5] E. Schrödinger, "Der stretige ubergang von der mikro-zur makromechanik," Naturwissenschaften, vol. 14, pp. 664-666, 1926.

[6] R. J. Glauber, "Some notes on multiple-boson processes," Physical Review, vol. 84, no. 3, pp. 395-400, 1951.

[7] V. V. Dodonov, "'Nonclassical' states in quantum optics: a "squeezed" review of the first 75 years," Journal of Optics B, vol. 4, no. 1, pp. R1-R33, 2002.

[8] G. Iwata, "Non-Hermitian operators and eigenfunction expansions," Progress of Theoretical Physics, vol. 6, pp. 212-221, 1951.

[9] J. P. Gazeau, T. Garidi, E. Huguet, M. Lachiére-Rey, and J. Renaud, “Examples of Berezin-Toeplitz quantization: finite sets and unit interval," in Symmetry in Physics, vol. 34 of CRM Proceedings E Lecture Notes, pp. 67-76, American Mathematical Society, Providence, RI, USA, 2004.

[10] J. P. Gazeau, Coherent States in Quantum Physics, Wiley-VCH, Weinheim, Germany, 2009.

[11] F. T. Arecchi, E. Courtens, R. Gilmore, and H. Thomas, "Atomic coherent states in quantum optics," Physical Review A, vol. 6, no. 6, pp. 2211-2237, 1972.

[12] J. R. Klauder, "The action option and a Feynman quantization of spinor fields in terms of ordinary c-numbers," Annals of Physics, vol. 11, pp. 123-168, 1960.

[13] E. C. Titchmarsh, Introduction to the Theory of Fourier Integrals, Calderon Press, Oxford, UK, 1937.

[14] Y. Katznelson, An Introduction to Harmonic Analysis, John Wiley \& Sons, New York, NY, USA, 1968.

[15] R. Fabec and G. Ólafsson, "The continuous wavelet transform and symmetric spaces," Acta Applicandae Mathematicae, vol. 77, no. 1, pp. 41-69, 2003.

[16] G. E. Andrews, R. Askey, and R. Roy, Special Functions, Cambridge University Press, Cambridge, UK, 1999.

[17] G. N. Watson, Treatise on the Theory of Bessel Functions, Cambridge University Press, Cambridge, UK, 1958.

[18] N. Aronszajn, "Theory of Reproducing Kernels," Transactions of the American Mathematical Society, vol. 68, pp. 337-404, 1950.

[19] I. S. Gradshteyn and I. M. Ryzhik, Table of Integrals, Series, and Products, Academic Press, 7th edition, 2007.

[20] J. R. Higgins, Completeness and Basis Properties of sets of Special Functions, vol. 72 of Cambridge Tracts in Mathematics, Cambridge University Press, Cambridge, UK, 1977. 


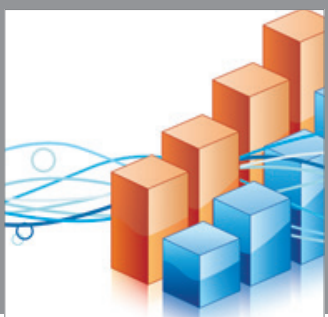

Advances in

Operations Research

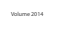

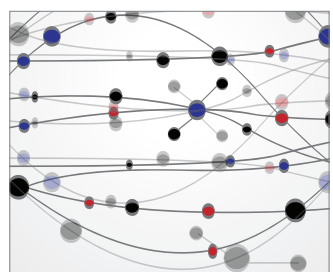

\section{The Scientific} World Journal
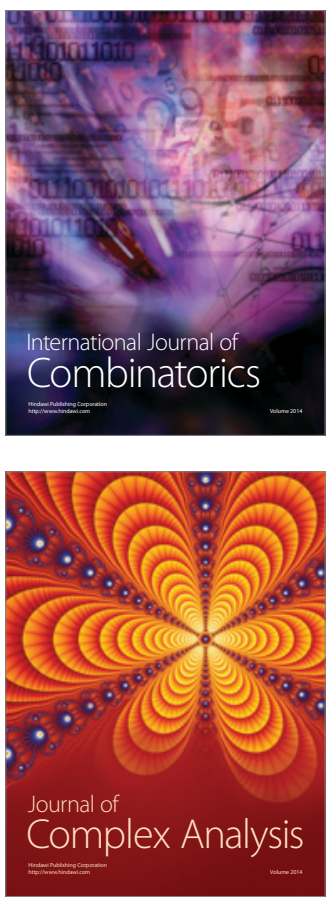

International Journal of

Mathematics and

Mathematical

Sciences
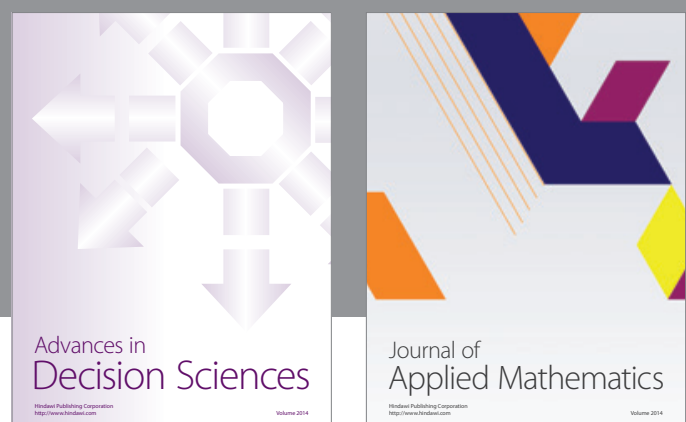

Journal of

Applied Mathematics
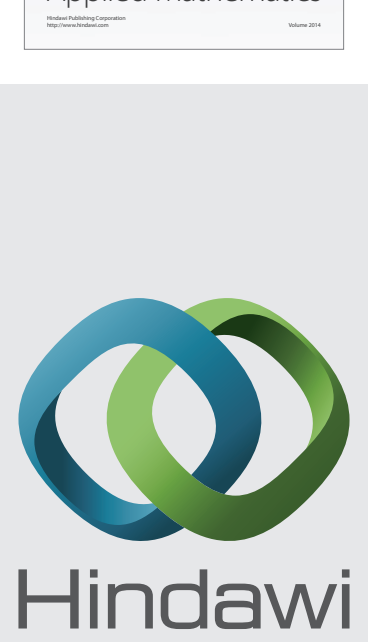

Submit your manuscripts at http://www.hindawi.com
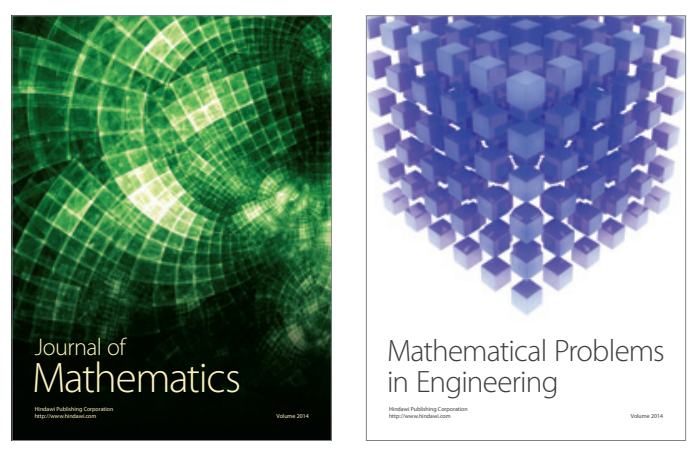

Mathematical Problems in Engineering
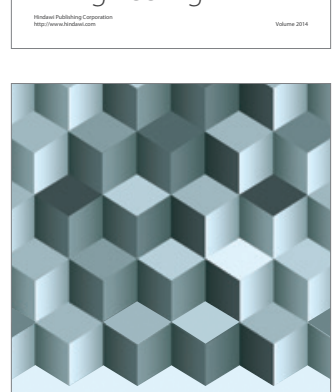

Journal of

Function Spaces
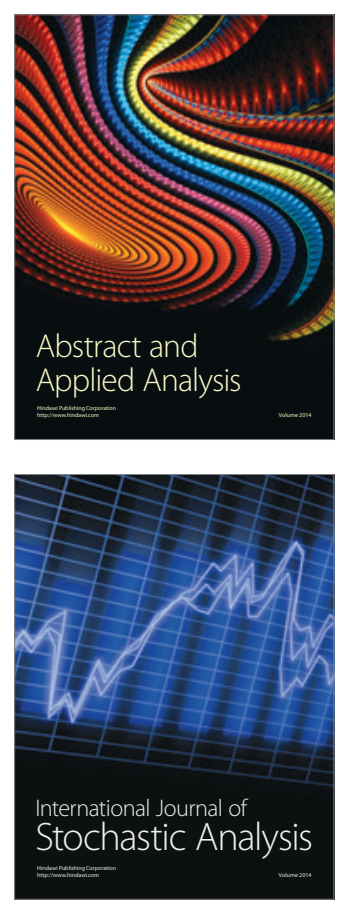

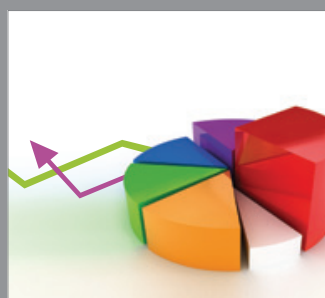

ournal of

Probability and Statistics

Promensencen
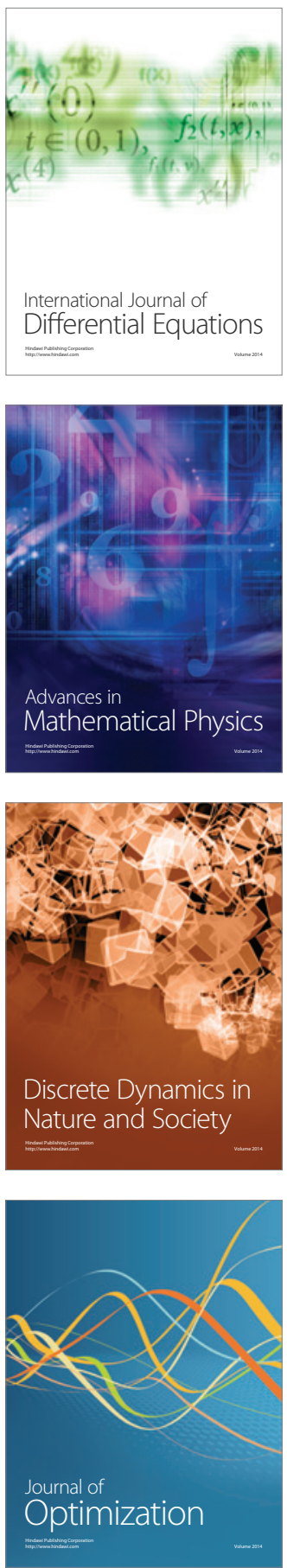